\title{
Clinical characteristics of people with cystic fibrosis and frequent fungal infection
}

\author{
Thomas Poore ${ }^{1}$, Maxene Meier ${ }^{2}$, Elin Towler ${ }^{2}$, John Brinton ${ }^{2}$, Stacey Martiniano ${ }^{3}$, Scott \\ Sagel $^{4}$, Emily DeBoer ${ }^{5}$, Brandie Wagner ${ }^{2}$, and Edith Zemanick ${ }^{6}$ \\ ${ }^{1}$ University of Colorado Denver School of Medicine \\ ${ }^{2}$ University of Colorado Denver - Anschutz Medical Campus \\ ${ }^{3}$ University of Colorado at Denver - Anschutz Medical Campus \\ ${ }^{4}$ University of Colorado Health Sciences Center \\ ${ }^{5}$ University of Colorado Anschutz Medical Campus \\ ${ }^{6}$ University of Colorado School of Medicine
}

June 21, 2021

\begin{abstract}
Background: Individuals with cystic fibrosis ( $\mathrm{CF}$ ) and fungal airway infection may present with fungal bronchitis, allergic bronchopulmonary aspergillosis (ABPA) or may appear unaffected despite fungal detection. We sought to characterize people with $\mathrm{CF}$ with frequent detection of fungi from airway samples and determine clinical outcomes. Methods: This retrospective study included individuals with CF with [?] 4 lower airway cultures over a 2-year baseline period and [?] 2 years of follow-up. We defined two groups: [?] 1 positive fungus culture (rare) or [?] 2 positive cultures during baseline (frequent). Clinical characteristics and outcomes were determined. Results: Between 2004-2016, 294 individuals met inclusion with 62\% classified as rare and $38 \%$ as frequent fungi during baseline. Median follow-up was 6 years (range 2-9 years). Aspergillus fumigatus was the most common fungal species detected. Individuals with frequent fungi were older (13.7 vs. $11.7 \mathrm{yrs}, \mathrm{p}=0.02)$ and more likely to have Stenotrophomonas maltophilia (35\% vs $17 \%, \mathrm{p}<0.001)$ at baseline, but did not differ in lung function or ABPA diagnosis. During follow-up, those with frequent fungi were more likely to have chronic P. aeruginosa and S. maltophilia. Individuals with ABPA and frequent fungi had the highest rates of co-infection and co-morbidities, and a trend towards more rapid lung function decline. Discussion: Fungal infection in CF was associated with frequent P. aeruginosa and S. maltophilia co-infection even in those without ABPA. Individuals with frequent fungi and ABPA had worse outcomes, highlighting the potential contribution of fungi to CF pulmonary disease.
\end{abstract}

Title: Clinical characteristics of people with cystic fibrosis and frequent fungal infection

Authors:

T. Spencer Poore, $\mathrm{MD}^{1,3}$, Maxene Meier, $\mathrm{MS}^{1,2}$, Elinor Towler, $\mathrm{MS}^{1,3}$, Stacey L. Martiniano, MD, MSCS ${ }^{1,3}$, John T. Brinton, Ph.D ${ }^{1,2}$, Emily M. DeBoer, MD, MSCS $^{1,3}$, Scott D. Sagel, MD, Ph.D ${ }^{1,3}$, Brandie D. Wagner, Ph.D, ${ }^{1,2}$, Edith T. Zemanick, MD, MSCS ${ }^{1,3}$

\section{Affiliations}

${ }^{1}$ University of Colorado, Anschutz Medical Campus, Department of Pediatrics

${ }^{2}$ University of Colorado, Anschutz Medical Campus, Department of Biostatistics and Informatics, Aurora, Colorado, USA 
${ }^{3}$ Section of Pediatric Pulmonary and Sleep Medicine and Children's Hospital Colorado, Aurora, Colorado, USA

Keywords: Aspergillus, Fungus, Infection, Allergic Bronchopulmonary Aspergillosis, Allergy

Funding: This project was supported by funding from the Cystic Fibrosis Foundation POORE20D0 (TSP), ZEMANI17Y5 (ETZ), ZEMANI20Y7 (ETZ, MM), NIH/NCATS Colorado CTSA Grant Number UL1 TR002535.

\section{Conflicts of Interests :}

ETZ personal fees from Cystic Fibrosis Foundation, site clinical trial support from Vertex Pharmaceuticals and Savara Pharmaceuticals Inc., and material agreements and consulting contracts with Calithera Biosciences and Concert Pharmaceuticals outside the scope of the submitted work. EMD is a consultant and stockholder for EvoEndoscopy, not related to this project.

Author Contributions : TSP, ETZ conceived of and designed the analysis and wrote the paper; MM performed the analysis and contributed to writing the paper; ET collected and provided the data; SM, ED, SS contributed to the study design, data analysis and critically reviewed the paper; BDW, JTB contributed to the data analysis and critically reviewed the paper.

\section{Abstract}

Background: Individuals with cystic fibrosis $(\mathrm{CF})$ and fungal airway infection may present with fungal bronchitis, allergic bronchopulmonary aspergillosis (ABPA) or may appear unaffected despite fungal detection. We sought to characterize people with $\mathrm{CF}$ with frequent detection of fungi from airway samples and determine clinical outcomes.

Methods: This retrospective study included individuals with CF with [?] 4 lower airway cultures over a 2-year baseline period and [?] 2 years of follow-up. We defined two groups: [?] 1 positive fungus culture (rare) or [?] 2 positive cultures during baseline (frequent). Clinical characteristics and outcomes were determined.

Results: Between 2004-2016, 294 individuals met inclusion with $62 \%$ classified as rare and $38 \%$ as frequent fungi during baseline. Median follow-up was 6 years (range 2-9 years). Aspergillusfumigatus was the most common fungal species detected. Individuals with frequent fungi were older (13.7 vs. $11.7 \mathrm{yrs}, \mathrm{p}=0.02)$ and more likely to have Stenotrophomonas maltophilia $(35 \%$ vs $17 \%$, p < 0.001$)$ at baseline, but did not differ in lung function or ABPA diagnosis. During follow-up, those with frequent fungi were more likely to have chronic P. aeruginosa and S.maltophilia. Individuals with ABPA and frequent fungi had the highest rates of co-infection and co-morbidities, and a trend towards more rapid lung function decline.

Discussion : Fungal infection in CF was associated with frequent $P$. aeruginosa and S. maltophilia coinfection even in those without ABPA. Individuals with frequent fungi and ABPA had worse outcomes, highlighting the potential contribution of fungi to CF pulmonary disease.

Introduction

Cystic fibrosis (CF) is a rare autosomal recessive disorder that affects more than 85,000 individuals worldwide. ${ }^{1}$ Despite significant advances in therapy, people with $\mathrm{CF}$ continue to experience high rates of morbidity and mortality due to pulmonary disease. ${ }^{2}$ People with CF are prone to inspissated airway mucus with subsequent pulmonary endobronchitis caused by cystic fibrosis transmembrane conductance regulator (CFTR ) protein dysfunction that impairs chloride and bicarbonate secretion at the airway surface layer. Individuals subsequently develop chronic airway infections, episodic pulmonary exacerbations, and progressive airway injury. ${ }^{3,4}$ Bacterial pathogens such asPseudomonas aeruginosa are well known contributors to disease progression and exacerbations. Fungi are also often isolated from lower airway samples [sputum and bronchoalveolar lavage fluid (BAL)] but their clinical impact is less well understood, especially when cultured in the presence of concomitant bacteria. ${ }^{5}$ 
Fungal infection in people with CF has a wide spectrum of both detection frequency and disease manifestations. Fungal detection may be transient, intermittent, or chronic. Disease manifestations range from acute or chronic fungal bronchitis with pulmonary exacerbation; sensitization to fungal allergens; and allergic bronchopulmonary mycosis (ABPM) most often caused by aspergillosis (allergic bronchopulmonary aspergillosis, ABPA). ${ }^{5-9}$ Others with fungal positive cultures may appear unaffected without worsening clinical signs or symptoms, or with symptoms attributable to other causes. ABPA is at the severe end of presentations and is a known allergic inflammatory process in reaction to Aspergillus antigen causing airway mucus impaction and obstruction.

Despite the possible associations, detection of fungus is not a requirement for an ABPA diagnosis. ${ }^{6,10,11}$ The impact of persistent fungal infection in the absence of ABPA on disease progression is less clear. Some studies have shown decrease in lung function over time in people with CF and Aspergillus infection as well as other worsening clinical outcomes. ${ }^{7}$ Other studies, however, have shown little clinical difference in those with or without fungi. ${ }^{12}$ Fungal detection has also been associated with $P$. aeruginosa co-infection and with inhaled antibiotic treatments often used to treat Pseudomonas, possibly due to changes in microbial competition within the community. ${ }^{13,14}$ Despite these studies, the contribution of fungi to CF lung disease progression remains under debate with few clinical guidelines upon isolation. Thus, we conducted a retrospective cohort study to compare clinical outcomes and disease course between individuals with CF with and without frequent fungal detection from airway samples, while accounting for chronic $P$. aeruginosa infection and ABPA diagnosis.

\section{Materials and Methods}

Patient selection and clinical characteristics: People with CF seen at Children's Hospital Colorado (CHCO) from 2004-2016 were identified by electronic medical records. Individuals were included in the study if they had a diagnosis of CF (sweat chloride [?] $60 \mathrm{mmol} / \mathrm{L}$ and/or two disease-causing CFTR gene mutations) as established by Cystic Fibrosis Foundation guidelines and at least 4 airway fungal cultures (sputum, $\mathrm{BAL}$, and/or fungus positive oropharyngeal (OP) culture) over two consecutive years, and at least 2 years of clinical data available after baseline. ${ }^{15}$ Information included was patient demographics, CF diagnostic criteria, genotype, comorbidities including ABPA, asthma, and CF related diabetes (CFRD) present during the baseline or follow-up periods, lung function, growth parameters, microbiology culture results, and laboratory results for IgE and complete blood count with eosinophils. Microbiologic culture data prior to baseline period was captured starting in 2004. Lung function results available from children 6 years and older were recorded.CFTR modulators prescribed were recorded, however availability of approved modulators was limited during the study time. The study was approved by the Colorado Multiple IRB (\#15-2376).

Cultures : Airway samples were collected as part of clinical care and processed at the CHCO clinical microbiology laboratory following standard CF culture procedures. Bacterial and fungal cultures are performed routinely on all sputum and BAL samples. ${ }^{16}$ Oropharyngeal cultures are not routinely plated on fungal media in our institution's microbiology lab, therefore result from oropharyngeal cultures were only included in the analysis if positive for fungi. Two or more positive cultures within one month of each other were considered a single positive culture. Fungal detection included any reported fungus species by our clinical lab, including the labels "yeast" and "fungus," which in some instances were reported in this manner.

Defining infection status: Initially we compared patients with no fungal positive cultures, rare fungus (one positive), intermittent fungi ([?] 2 positive but $<50 \%$ positive), or chronic fungi ( $>2$ positive and at least $50 \%$ positive); however, given the limited group sample sizes we chose to dichotomize fungal exposure to agree with previously published literature and to provide greater power for group comparisons. Results from cultures over the two-year baseline period were used to categorize individuals as either havingrare fungus , defined as [?] 1 positive fungal culture in the baseline period and no positive fungal cultures recorded prior to baseline, or frequent fungi, defined as having 2 or more positive fungal cultures in the baseline period. Definitions were based upon adaptations from previously published fungal and chronic bacterial infection studies in CF. ${ }^{7}, 17$ Given that we defined fungal exposure for individuals in the baseline period, individuals could transition groups during the follow-up period (e.g. those classified as rare could later meet 
critieria for frequent fungal infection). Participants were considered rare during follow-up if they had $\leq 1$ positive culture and considered frequent if they met criteria ( $\geq 2$ positive cultures) in any year of followup. Chronic P. aeruginosa status during the baseline follow-up period was defined by Leed's criteria of $>$ $50 \%$ positive cultures for $P$. aeruginosa during at least one year. Individuals who met criteria for chronic $P$. aeruginosa within any year during the follow-up period were considered chronically infected. ${ }^{18}$ Ever $P$. aeruginosa status during the follow up period was defined as isolating $P$. aeruginosa at any point in time, regardless of frequency or year.

\section{Statistical Analyses}

Summary descriptive statistics included means and standard deviations or median and range for continuous variables and frequencies and percentages for categorical data. Characteristics such as body mass index (BMI) that were repeated over the study period were averaged over their individual baseline and followup time. Differences between airway fungi groups were determined using ANOVAs or Chi-squared tests depending on the distribution. Given the potential impact of ABPA on clinical status, we also compared individuals with rare and frequent fungi by ABPA status, comparing characteristics and outcomes across the 4 groups. Multiple linear mixed effects regression models allowing for random slope and intercept per patient were used to assess percent predicted forced expiratory volume in one second (ppFEV1) over time by fungal exposure group using maximum likelihood estimation. ppFEV1 over follow-up time was standardized within patient by taking their average ppFEV1 during their baseline. The first model only included time and fungal exposure group, the second model adjusted for chronic P. aeruginosa and ABPA status, and the third and fourth models compared ppFEV1 by airway fungi and also adjusted for P. aeruginosastratified by ABPA status. Models were re-run adjusting for transition from one group to another in the follow up period to assess any change in association or inference. All three-way and two-way interactions between time with fungal status, ABPA, chronic or ever P. aeruginosa were assessed. Statistical significance was set at a Type I error rate of 0.05. All data cleaning, summaries, and testing were done using R (R Foundation for Statistical Computing, Vienna, Austria. URL https://www.R-project.org/).

Results

\section{Population characteristics during the baseline period}

During the study period, clinical data from 712 patients with CF were available in the clinical database and EMR, of whom 294 met inclusion criteria (Figure S1). At baseline, 113 patients (38\%) were categorized as having frequent fungi and 181 patients had rare fungus (62\%). Patient characteristics during the two-year baseline period are shown in Table 1. Aspergillus sp. was the most common fungus isolated in the baseline period for both the frequent and rare groups, with Aspergillus fumigatus being the most common. Individuals categorized as frequent fungi were older (median 13.7 versus 11.7 years, $\mathrm{p}=0.02$ ) and were more frequently co-infected with S. maltophilia $(35 \%$ versus $17 \%, \mathrm{p}<0.001)$ compared to those with rare fungus. We did not detect differences in baseline ppFEV1, BMI percentile or rates of ABPA between groups. Fewer individuals with frequent fungi were diagnosed with asthma compared to the rare fungus group $(14.9 \%$ versus $25.6 \%$, p $=0.04)$.

\section{Patient characteristics during the follow-up period}

Patient characteristics and clinical outcomes during follow-up period are shown in Table 2. There was no difference in the amount of follow-up time for those with frequent fungi compared to rare [mean (SD) 4.3 (2.4) versus $4.3(2.2)$ years, $\mathrm{p}=0.70]$. Those with frequent fungi had more respiratory cultures during this period compared to the rare group although the range was large [median (range) 10 (2-76) versus 7 (2-64) respectively, $\mathrm{p}=0.02]$. We did not detect differences in mean ppFEV1, BMI percentile, co-morbidities or IgE concentrations between groups. Individuals with frequent fungi were more likely to have chronic $P$. aeruginosa $(51 \%$ vs. $37 \%, \mathrm{p}=0.01)$ and to ever have cultured P. aeruginosa $(73 \%$ vs. $52 \%, \mathrm{p}<0.001)$ compared to those with rare fungus. Individuals with frequent fungi were also more likely to have S. maltophilia (47\% versus $30 \%, \mathrm{p}=0.004$ ) consistent with baseline findings. There were no differences observed in frequency of MRSA and MSSA between groups. 


\section{Relationship between change in fungal status and co-infections}

Of the individuals classifed as rare at baseline, $65(36 \%)$ subsequently had at least one year of frequent fungal infections during the study period, while $116(64 \%)$ remained rare throughout the study period. Of the individuals classifed as frequent at baseline, $25(22 \%)$ did not isolate fungus during the follow-up period. We examined co-infections with P. aeruginosa and S. maltophilia in patients who transitioned from rare to frequent fungi. Of the individuals who started in the rare group and transitioned to frequent $(\mathrm{n}=65), 17$ had $P$. aeruginosa positive cultures. In this group, $71 \%(12 / 17)$ had $P$. aeruginosa detected prior to fungal positive culture whereas $29 \%(5 / 17)$ had a positive fungal culture prior to $P$. aeruginosadetection. Of the 38 individuals with $S$. maltophilia, $45 \%$ (17/38) had S. maltophilia detected prior to fungal positive culture whereas $55 \%(21 / 38)$ had a positive fungal culture prior to $S$. maltophilia detection.

\section{Relationships of fungal status with changes in lung function}

Average rate of $\mathrm{ppFEV}_{1}$ decline during follow-up was not statistically different between the frequent fungi group compared to the rare group (-1.85\% versus $-1.61 \%$ per year respectively, figure S2). Using regression modeling, there was no difference between fungal groups, even when controlling for ABPA, chronic $P$. aeruginosa, and transitioning across groups. Interestingly, in this model, when comparing only those with $\mathrm{ABPA}$ and frequent fungal infection to those with $\mathrm{ABPA}$ and rare fungal infection, fungal status was significant, showing that those with ABPA and frequent fungal infection had lower lung function at each time point compared to those with ABPA and rare fungal infection $(\mathrm{p}=0.047)$.

\section{Individuals with ABPA and frequent fungal infection have signs of worse clinical outcomes}

We compared the clinical characteristics at baseline and during follow-up of individuals by ABPA and fungal status: $\mathrm{ABPA} /$ frequent $(20,7 \%), \mathrm{ABPA} /$ rare $(27,9 \%)$, no $\mathrm{ABPA} /$ frequent $(93,32 \%)$, and no $\mathrm{ABPA} /$ rare $(154,52 \%)$. Individuals ever diagnosed with ABPA were, on average, followed longer during the study period than those without ABPA regardless of fungal status [mean (SD) 5.7 yrs (2.3) versus 4.0 (2.7) yrs, respectively, $\mathrm{p}<0.001$. Table 3 shows clinical characteristics for the four groups. Figure 1 shows comparisons in $\mathrm{ppFEV}_{1}$, diagnoses of CFRD, IgE measurements, and co-infection with P. aeruginosa and S. maltophilia between groups.

Individuals with frequent fungi and ABPA tended to have more chronic infection with P. aeruginosa compared to the other three groups $(\mathrm{p}=0.07)$. While not statistically significant, those with frequent fungal infection and ABPA had the lowest average ppFEV1 of the four groups during the follow up period (Table 3 ) and highest annual rate of decline in ppFEV1 [ $-2.3 \%$ versus ABPA/Rare $-1.32 \%$, No ABPA/Frequent $-1.76 \%$ and No ABPA/Rare $-1.68 \%$ per year ( $\mathrm{p}$ values of interactions $=0.19,0.5,0.43$ ), Figure 2$]$. Those with ABPA had higher IgE and serum eosinophils regardless of fungal status; IgE was highest in those with $\mathrm{ABPA}$ and frequent fungal infections.

\section{Discussion}

In this large retrospective cohort study, we found that individuals with $\mathrm{CF}$ and frequent fungal detection had higher rates of co-infection with $P$. aeruginosa and S. maltophilia compared to those with no or rare detection of fungi. Other clinical characteristics and outcomes did not differ between those with frequent versus rare fungi including frequency of ABPA diagnosis, nutritional status, and rate of pulmonary exacerbations. Lung function $\left(\mathrm{ppFEV}_{1}\right)$ and lung function decline were also similar between groups including after controlling for chronic P. aeruginosa and ABPA.

In individuals with ABPA however, frequent fungal detection was associated with lower lung function $\left(\mathrm{ppFEV}_{1}\right)$ compared to those with ABPA but rare fungal detection. Individuals with ABPA/frequent fungi had higher rates of co-infection with $P$. aeruginosa and $S$. maltophilia ; more CFRD diagnoses; and higher IgE values compared to those with $\mathrm{ABPA} /$ rare fungi and compared to those without ABPA regardless of fungal categorization. Individuals with ABPA/ frequent fungi also had a trend towards more rapid lung function decline during follow-up. Interestingly, we found equal proportions of ABPA among the rare and frequent fungal groups. 
As expected, those with ABPA had higher absolute eosinophils and IgE values, markers of T-helper cells type 2 (Th2), typical of allergic or hypersensitivity responses. We did not detect a difference in eosinophils or IgE between the frequent and rare fungal groups. Interestingly, $60 \%$ of the rare group and $55 \%$ of the frequent group had a diagnosis of asthma over the entire study, which is higher than the general population as well as previously published literature on patients with CF. ${ }^{19,}{ }^{20}$ Furthermore, in the baseline period, more individuals in the rare group had asthma, while in the follow up period, more individuals in the frequent group had asthma. . While the follow up period had more time to collect data and is likely more robust, the significance of this finding is unclear. Asthma diagnoses were documented based upon coding in our electronic medical record; thus diagnoses may reflect institutional practices, regional effects and allergens, or another unknown confounding variable.

Individuals with frequent fungal infection had higher rates of chronic $P$. aeruginosa co-infection and more $P$. aeruginosadetection overall compared to the rare fungus group. A similar pattern was seen in those with ABPA and frequent fungi, having the highest proportion of individuals with chronic P. aeruginosa infection when compared to the other three groups. This may be related to the use of aggressive antibiotic therapy in $P$. aeruginosa eradication protocols, allowing for shifts in biodiversity by creating a less competitive airway environment for fungal species. ${ }^{11,21}$ Other studies and authors have suggested that biofilms created by $P$. aeruginosa and/or Aspergillus may be initially favorable and synergistic for fungal spores to germinate. ${ }^{22}$ However, other studies have shown that $P$. aeruginosa biofilms actually inhibit Aspergillusgrowth, favoring the competition theory. ${ }^{23}$ There was no significant differences in MRSA co-infection among any of the groups, another organism possibly related to more frequent therapies and driven by microbial management. ${ }^{24}$ In the follow-up period, people in our cohort with rare fungi more often isolated $P$. aeruginosa prior to later fungal detection, rather than isolating fungi first, supporting the role of antimicrobial therapies and/or a synergistic environment in fungal growth, although our numbers were small.

S. maltophilia was associated with more frequent fungal infection at baseline and during follow-up. It also had a different longitudinal pattern than P. aeruginosa, showing that almost $50 \%$ isolated S. maltophilia prior to fungus and vice versa. While one investigation in the associations with fungi and S. maltophiliahave been seen in people with CF and liver disease, the literature is still lacking in understanding of how the organisms are related. ${ }^{25}$ Our findings are both supportive of and contrary to existing data, but imply an association between the acquisition of fungus and subsequent infection with P. aeruginosaand S. maltophilia . Given this, more research is needed to identify associated risk factors as well as cause and effect relationships between these organisms.

The association between fungus and lung disease progression has been examined previously in CF. Studies have shown lower ppFEV1 in those with chronic fungal infection, yet the definition of chronic varies widely in the literature. Some investigators have adapted criteria similar to that of $P$. aeruginosa classifications, however there are many genera and species of fungus that make definitions difficult. Furthermore, given the need for sputum or BAL for fungal culture, detection of fungi is dependent on the person's ability to expectorate, further complicating classification, prevalence, and disease severity. This likely explains the higher rates of bacteria and fungus isolation in our cohort when compared to registry data. ${ }^{26}$

Most studies in the literature focus on Aspergillus or Aspergillus fumigatus specifically, excluding other fungal genera. $^{5,7,17}$ We included all fungi (with the exception of Candida that is not routinely reported by our clinical laboratory) as genera such as Scedosporium may also contribute to CF lung disease. ${ }^{27}$, ${ }^{28}$ However, over $70 \%$ of fungal positive cultures in our cohort grew Aspergillus . Our study was larger than most and included follow-up for a median of 6 years.

Our findings agree with the literature in finding higher morbidity and worse outcomes in those with ABPA. However, our study was one of the few comparing ABPA to fungal frequency both as a co-variate and as a separate group. ${ }^{6,7}$ By doing so, we found that those with both ABPA and frequent fungus appeared to have more severe disease despite the fact that the presence of fungus in the sputum is not required for ABPA diagnosis. This dynamic emphasizes the importance of fungus on inflammation in the CF airway, yet how the pathophysiology of fungal infections needs further investigation. 
This study has several limitations. Most importantly, this was a single center, retrospective study so we are unable to determine causation between fungal disease, ABPA and outcomes. Given electronic medical record limitations, we only evaluated culture results as early as 2004. It is possible that some individuals isolated fungi before this and thus meet other fungal group classification. Fungi are also difficult to isolate in culture. ${ }^{21,}{ }^{29}$ Given this, there are likely individuals who have fungi that were not detected and may account for those without ABPA in the absence of positive fungal cultures. We tried to account for this by requiring at least 4 lower airway cultures for analysis. It is likely that most of the individuals in this study could spontaneously expectorate, and thus were likely more severely impacted compared to those who could not expectorate. This may explain the higher rates of fungi and bacterial pathogens given a more reliable sample than throat swabs. Furthermore, individuals in the frequent fungal group had significantly more cultures compared to those with rare fungi which may lead to surveillance bias. Prospective longitudinal studies are needed to assess the acquisition of fungus in real time and how use of antibiotics and co-infections contribute. Mechanistic studies are also needed to explore the interactions of fungi and other organisms in the CF airway as well as inflammatory host-responses, specific biomarkers of fungal disease and allergy, and clinical studies investigating characteristics regarding the development of ABPA.

Conclusions

We found associations between frequent fungal detection and bacterial co-infection with P. aeruginosa and S. maltophilia, but not MSSA or MRSA in people with CF. In those with a diagnosis of ABPA, frequent fungal detection was associated with worse clinical outcomes with high rates of comorbidities, co-infections with pathogenic bacteria, and worse lung disease. However, we did not detect a similar association between fungal detection and clinical outcomes when controlling for ABPA diagnosis. Future prospective studies are needed to determine the relationship between fungal infection, bacterial co-infections and development of ABPA.

\section{References}

[1] Bell SC, Mall MA, Gutierrez H, Macek M, Madge S, Davies JC, Burgel PR, Tullis E, Castanos C, Castellani C, Byrnes CA, Cathcart F, Chotirmall SH, Cosgriff R, Eichler I, Fajac I, Goss CH, Drevinek P, Farrell PM, Gravelle AM, Havermans T, Mayer-Hamblett N, Kashirskaya N, Kerem E, Mathew JL, McKone EF, Naehrlich L, Nasr SZ, Oates GR, O’Neill C, Pypops U, Raraigh KS, Rowe SM, Southern KW, Sivam S, Stephenson AL, Zampoli M, Ratjen F: The future of cystic fibrosis care: a global perspective. Lancet Respir Med 2020, 8:65-124.

[2] Elborn JS: Cystic fibrosis. Lancet 2016, 388:2519-31.

[3] Gibson RL, Burns JL, Ramsey BW: Pathophysiology and management of pulmonary infections in cystic fibrosis. Am J Respir Crit Care Med 2003, 168:918-51.

[4] Stoltz DA, Meyerholz DK, Welsh MJ: Origins of cystic fibrosis lung disease. N Engl J Med 2015, $372: 1574-5$.

[5] Tracy MC, Moss RB: The myriad challenges of respiratory fungal infection in cystic fibrosis. Pediatr Pulmonol 2018, 53:S75-S85.

[6] Janahi IA, Rehman A, Al-Naimi AR: Allergic bronchopulmonary aspergillosis in patients with cystic fibrosis. Ann Thorac Med 2017, 12:74-82.

[7] Amin R, Dupuis A, Aaron SD, Ratjen F: The effect of chronic infection with Aspergillus fumigatus on lung function and hospitalization in patients with cystic fibrosis. Chest 2010, 137:171-6.

[8] Baxter CG, Dunn G, Jones AM, Webb K, Gore R, Richardson MD, Denning DW: Novel immunologic classification of aspergillosis in adult cystic fibrosis. J Allergy Clin Immunol 2013, 132:560-6 e10.

[9] King J, Brunel SF, Warris A: Aspergillus infections in cystic fibrosis. J Infect 2016, 72 Suppl:S50-5. 
[10] Antunes J, Fernandes A, Borrego LM, Leiria-Pinto P, Cavaco J: Cystic fibrosis, atopy, asthma and ABPA. Allergol Immunopathol (Madr) 2010, 38:278-84.

[11] Sudfeld CR, Dasenbrook EC, Merz WG, Carroll KC, Boyle MP: Prevalence and risk factors for recovery of filamentous fungi in individuals with cystic fibrosis. J Cyst Fibros 2010, 9:110-6.

[12] de Vrankrijker AM, van der Ent CK, van Berkhout FT, Stellato RK, Willems RJ, Bonten MJ, Wolfs TF: Aspergillus fumigatus colonization in cystic fibrosis: implications for lung function? Clin Microbiol Infect 2011, 17:1381-6.

[13] Burns JL, Van Dalfsen JM, Shawar RM, Otto KL, Garber RL, Quan JM, Montgomery AB, Albers GM, Ramsey BW, Smith AL: Effect of chronic intermittent administration of inhaled tobramycin on respiratory microbial flora in patients with cystic fibrosis. J Infect Dis 1999, 179:1190-6.

[14] Keown K, Reid A, Moore JE, Taggart CC, Downey DG: Coinfection with Pseudomonas aeruginosa and Aspergillus fumigatus in cystic fibrosis. Eur Respir Rev 2020, 29.

[15] Farrell PM, White TB, Ren CL, Hempstead SE, Accurso F, Derichs N, Howenstine M, McColley SA, Rock M, Rosenfeld M, Sermet-Gaudelus I, Southern KW, Marshall BC, Sosnay PR: Diagnosis of Cystic Fibrosis: Consensus Guidelines from the Cystic Fibrosis Foundation. J Pediatr 2017, 181S:S4-S15 e1.

[16] Saiman L, Siegel J, Cystic Fibrosis F: Infection control recommendations for patients with cystic fibrosis: microbiology, important pathogens, and infection control practices to prevent patient-to-patient transmission. Infect Control Hosp Epidemiol 2003, 24:S6-52.

[17] Heltshe SL, Mayer-Hamblett N, Burns JL, Khan U, Baines A, Ramsey BW, Rowe SM, Network GIotCFFTD: Pseudomonas aeruginosa in cystic fibrosis patients with G551D-CFTR treated with ivacaftor. Clin Infect Dis 2015, 60:703-12.

[18] Lee TW, Brownlee KG, Conway SP, Denton M, Littlewood JM: Evaluation of a new definition for chronic Pseudomonas aeruginosa infection in cystic fibrosis patients. J Cyst Fibros 2003, 2:29-34.

[19] Kent BD, Lane SJ, van Beek EJ, Dodd JD, Costello RW, Tiddens HA: Asthma and cystic fibrosis: a tangled web. Pediatr Pulmonol 2014, 49:205-13.

[20] Nielsen AO, Qayum S, Bouchelouche PN, Laursen LC, Dahl R, Dahl M: Risk of asthma in heterozygous carriers for cystic fibrosis: A meta-analysis. J Cyst Fibros 2016, 15:563-7.

[21] Nguyen LD, Viscogliosi E, Delhaes L: The lung mycobiome: an emerging field of the human respiratory microbiome. Front Microbiol 2015, 6:89.

[22] Kaur S, Singh S: Biofilm formation by Aspergillus fumigatus. Med Mycol 2014, 52:2-9.

[23] Mowat E, Rajendran R, Williams C, McCulloch E, Jones B, Lang S, Ramage G: Pseudomonas aeruginosa and their small diffusible extracellular molecules inhibit Aspergillus fumigatus biofilm formation. FEMS Microbiol Lett 2010, 313:96-102.

[24] Akil N, Muhlebach MS: Biology and management of methicillin resistant Staphylococcus aureus in cystic fibrosis. Pediatr Pulmonol 2018, 53:S64-S74.

[25] Cabaret O, Bonnal C, Canoui-Poitrine F, Emirian A, Bizouard G, Levesque E, Maitre B, Fihman V, Decousser JW, Botterel F: Concomitant presence of Aspergillus fumigatus and Stenotrophomonas maltophilia in the respiratory tract: a new risk for patients with liver disease? J Med Microbiol 2016, 65:414-9.

[26] Foundation CF: 2019 Patient Registry Annual Data Report. 2020.

[27] Cimon B, Carrere J, Vinatier JF, Chazalette JP, Chabasse D, Bouchara JP: Clinical significance of Scedosporium apiospermum in patients with cystic fibrosis. Eur J Clin Microbiol Infect Dis 2000, 19:53-6. 
[28] Chotirmall SH, McElvaney NG: Fungi in the cystic fibrosis lung: bystanders or pathogens? Int J Biochem Cell Biol 2014, 52:161-73.

[29] Coron N, Pihet M, Frealle E, Lemeille Y, Pinel C, Pelloux H, Gargala G, Favennec L, Accoceberry I, Durand-Joly I, Dalle F, Huet F, Fanton A, Boldron A, Loeuille GA, Domblides P, Coltey B, Pin I, Llerena C, Troussier F, Person C, Marguet C, Wizla N, Thumerelle C, Turck D, Bui S, Fayon M, Duhamel A, Prevotat A, Wallaert B, Leroy S, Bouchara JP, Delhaes L: Toward the Standardization of Mycological Examination of Sputum Samples in Cystic Fibrosis: Results from a French Multicenter Prospective Study. Mycopathologia 2018, 183:101-17.

\section{Hosted file}

Tables Only2.docx available at https://authorea.com/users/420923/articles/527139-clinicalcharacteristics-of-people-with-cystic-fibrosis-and-frequent-fungal-infection
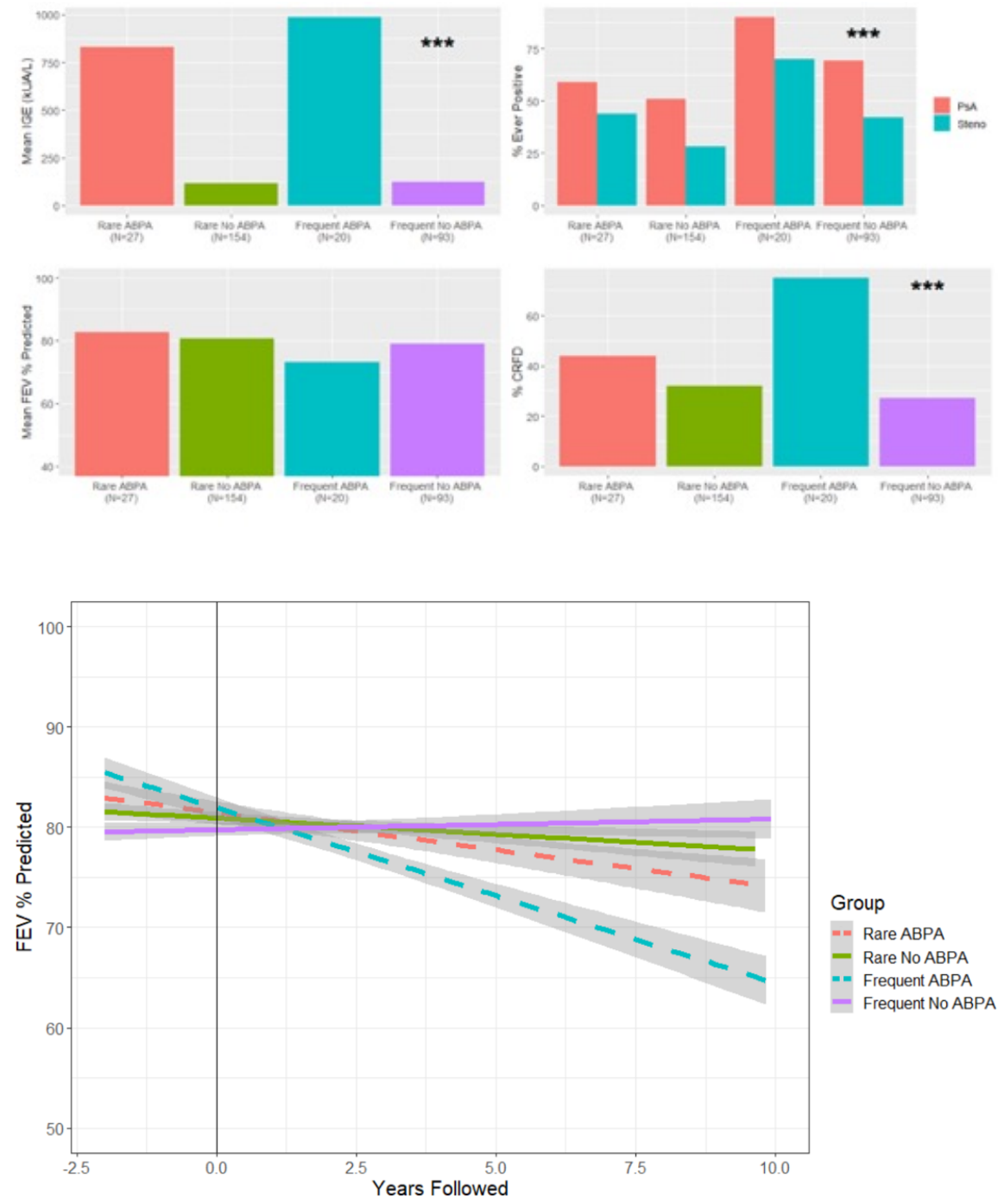